\title{
Cotyledonary node pre-wounding with a micro-brush increased frequency of Agrobacterium-mediated transformation in soybean
}

\author{
Tetsuya Yamada ${ }^{1, *}$, Satoshi Watanabe ${ }^{2}$, Maiko Arai ${ }^{1}$, Kyuya Harada ${ }^{2}$, Keisuke Kitamura ${ }^{1}$ \\ ${ }^{1}$ Graduate School of Agriculture, Hokkaido University, Sapporo, Hokkaido 060-8589, Japan; ${ }^{2}$ National Institute of \\ Agrobiological Sciences, Tsukuba, Ibaraki 305-8602, Japan \\ *E-mail: tetsuyay@res.agr.hokudai.ac.jp TEL: +81-11-706-4186 FAX: +81-11-706-4933
}

Received December 18, 2009; accepted April 2, 2010 (Edited by T. Anai)

\begin{abstract}
A Japanese soybean variety, Kariyutaka, known to form many adventitious shoots from sections of the cotyledonary node, had a transformation frequency (relative number of $\mathrm{T}_{0}$ transgenic plants, which stably transmitted transgenes to their $\mathrm{T}_{1}$ progenies, to number of explants subjected to Agrobacterium-infection) of about $1.0 \%$ in the conventional protocol. We modified this method to obtain the transformation frequency of $4.4 \%$. Modified protocol included mechanical wounding of explants with a micro-brush and application of $0.02 \%$ of Silwet L-77 during the infection with Agrobacterium. We obtained $\mathrm{T}_{0}$ plants 8 to 12 weeks after co-cultivation with Agrobacterium. $\mathrm{T}_{1}$ seeds were rapidly obtained from 5 to 6 months after the co-cultivation, for $\mathrm{T}_{0}$ plants flowered precociously. Three $\mathrm{T}_{0}$ plants out of 4 , i.e. $75 \%$ of all $\mathrm{T}_{0}$ plants, which were generated at 12 weeks after co-cultivation, possessed stable transgene(s) that were transmitted to their $\mathrm{T}_{1}$ progenies, although only $7 \mathrm{~T}_{0}$ plants out of 22 , i.e. $32 \%$ of all $\mathrm{T}_{0}$ plants, which were generated at 8 weeks after the co-cultivation, stably transmitted the Basta-resistance to their $\mathrm{T}_{1}$ progenies.
\end{abstract}

Key words: Glycine max, Japanese variety, rapid life cycle, stable transformation.

A large amount of sequences which is derived from the soybean genome can be retrieved from Phytozome (http://www.phytozome.net/soybean). The information is useful to isolate gene homologues or predict candidate genes for the map-based cloning. An efficient and stable transformation technology is required to identify the function of the isolated gene homologues. Currently, there are two major transformation methods, particle bombardment and Agrobacterium-infection. The latter offers several advantages such as few fragmentations of inserted T-DNA regions, and stable expression and low copy number of integrated transgenes (Birch 1997). It uses Agrobacterium as a biological vector for DNA delivery and needs regenerative adventitious shoots from the cotyledonary node of soybean seedlings (Hinchee et al. 1988). The transformation frequency by this method in soybeans still remains very low (Dinkins and Collins 2008). In the processes of infection and co-cultivation with Agrobacterium, there are some critical steps to conduct stable and efficient transformation. The conditions for infection with Agrobacterium and cocultivation were optimized by application of a surfactant (Silwet L-77) to the bacterial suspension, and maintenance of the culture temperature and $\mathrm{pH}$ of the medium (Dang and Wei 2007; Liu et al. 2008). To enhance bacterial delivery, explants were sonicated in the presence of Agrobacterium for a short time (Meurer et al. 1998; Trick and Finer 1997). Recently, sections of the cotyledonary node on explants have been wounded mechanically with a scalpel or a small needle to enhance Agrobacterium infection (Olhoft et al. 2001; Xue et al. 2006). Therefore, modifying infection and co-cultivation with Agrobacterium might improve efficiency of transgenic soybean plant generation.

We found that a Japanese soybean variety, Kariyutaka, possessed advantages for Agrobacterium-mediated transformation, such as the production of many adventitious shoots, vigorous shoot elongation, and prolific rooting (Sato et al. 2007). However, a transformation frequency in this variety was only approximately $1.0 \%$ (Sato et al. 2007), which was defined as the relative number of $\mathrm{T}_{0}$ plants transmitting transgenes to their progenies to number of Agrobacterium-infected explants, and this rate was not high enough to obtain stable transgenic plants. In this study, we describe an improved protocol of Agrobacterium-mediated transformation of soybean, with modification of using the cotyledonary node that was wounded with a micro-brush. The transformation frequency in the method presented here was 4 times as high as in that obtained with our previous method (Sato et al. 2007).

Kariyutaka, which was also currently available as a resource (JP 86520) from Genebank, National Institute

This article can be found at http://www.jspcmb.jp/ 
of Agrobiological Science, was obtained from Hokkaido Prefectural Tokachi Agricultural Experiment Station. The frequency of soybean transformation was evaluated using a plasmid vector, pMDC123-IG, which was constructed from pMDC123-GFP (Sato et al. 2007) by replacing the sGFP gene (Niwa et al. 1999) with the intron GUS gene (Ohta et al. 1990). The original pMDC123 vector (Curtis and Grossniklaus 2003) contains the Bar gene as a selection marker with glufosinate-ammonium (Sigma-Aldrich) or Basta ${ }^{\circledR}$ (Bayer Crop Science).

Agrobacterium-mediated transformation of soybean was conducted as described by Sato et al. (2007) with four major modifications. Sterilized soybean seeds were transferred on a plate and maintained in a plastic container filled with sterilized water for one week at $26^{\circ} \mathrm{C}$ to adjust the water content of the seeds. The cotyledonary node of each explant was wounded by scratching with a stainless steel micro-brush (AS ONE, MBRS-02) (Figure 1A). The wounded explants were dipped in the Agrobacterium suspension with the cocultivation medium containing $0.02 \%$ of Silwet L-77 (Bio Medical Science). Elongating adventitious shoots were maintained in a rooting medium containing $6 \mathrm{mgl}^{-1}$ glufosinate-ammonium for 2 weeks prior to transfer to another rooting medium without the selectable agent. The transgenic plants were assessed on the basis of a histochemical $\beta$-glucuronidase assay (GUS assay) as described by Sato et al. (2007).

For Agrobacterium-mediated transformation, explants containing the cotyledonary node section were excised from seedlings 5-7 days old (Olhoft et al. 2001; Zeng et al. 2004). Recently, mature seeds which were preserved over desiccants (silica gel) at $4^{\circ} \mathrm{C}$ were subjected to overnight imbibition, and were used for soybean transformation (Paz et al. 2006). In a previous study, we also used mature seeds that were pretreated in this way as an explant for the transformation experiment (Sato et al. 2007). In order to prevent generation of cracks or collapse in seeds upon sudden imbibition, we maintained mature seeds in a humid plastic container to increase seed water content gradually. This pretreatment increased the water content of the seeds from 5.6 $( \pm 2.1) \quad \%$ to $29.1 \quad( \pm 1.2) \quad \%$ after the 1-week incubation period. In the previous study, we used explants for Agrobacterium-mediated transformation without wounding the cotyledonary node sections and employing a method described by Paz et al. (2006). In this study, the cotyledonary node of the explants was wounded using a micro-brush to enhance the infection with Agrobacterium (Figure 1A). We applied 0, 5, and 10 times of scratching on the cotyledonary node section before the infection. The more scratches were applied, the more prominent GUS expression was (Figure 1B-D). Application of a surfactant to the Agrobacterium
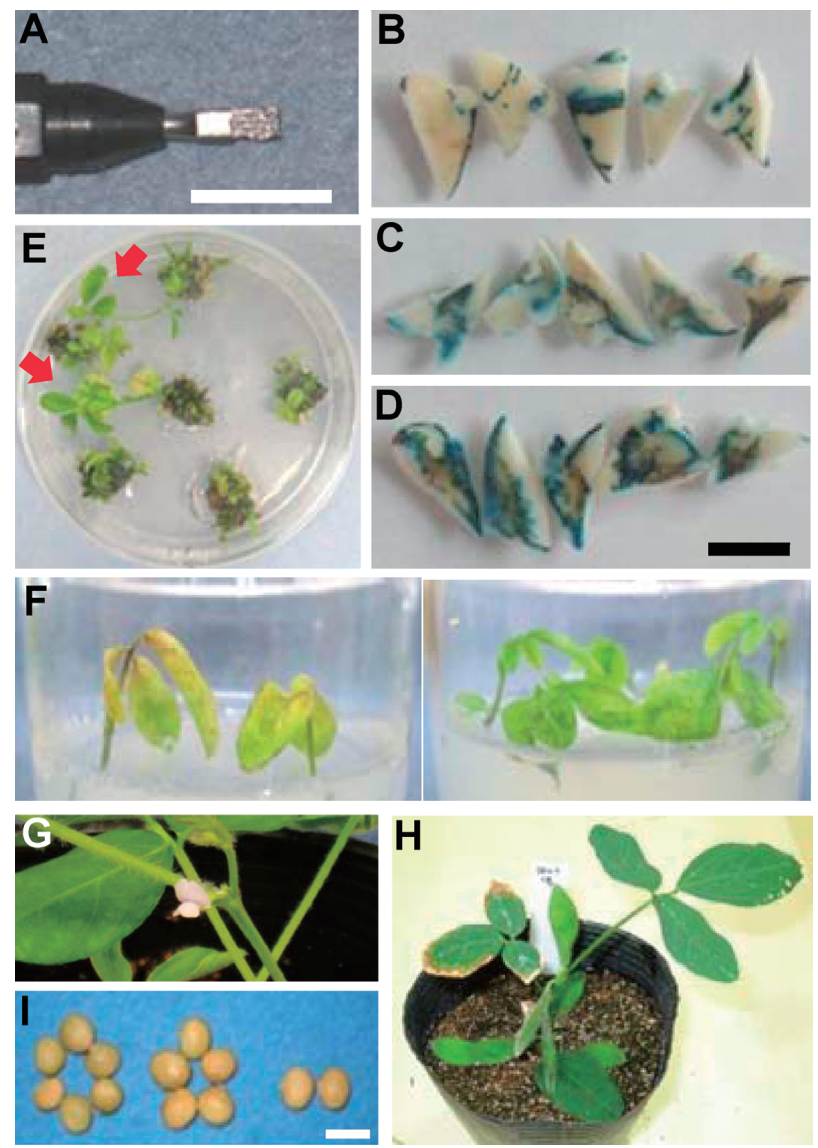

Figure 1. Agrobacterium-mediated transformation by using the mechanically wounded cotyledonary node. (A) Stainless-made microbrush for scratching cotyledonary nodes. (B-D) Histochemical GUS assay of explants without wounding (B), with by 5 scratches (C), and with by 10 scratches (D). (E) Adventitious shoots elongating from explants with 10 scratches. Red arrows indicate elongating shoots on the shoot elongation medium containing $6 \mathrm{mgl}^{-1}$ glufosinateammonium. (F) Selection of transgenic shoots on the rooting medium containing $6 \mathrm{mg} \mathrm{l}^{-1}$ glufosinate-ammonium. Sensitive (left) and resistant (right) shoots to glufosinate-ammonium selection. (G) A precocious flower in a young $\mathrm{T}_{0}$ plant. (H) $A \mathrm{~T}_{0}$ plant which set pods. (I) Mature $\mathrm{T}_{1}$ seeds obtained from 3 independent $\mathrm{T}_{0}$ plants. Seeds from each $\mathrm{T}_{0}$ plant were grouped. Scale bars represent $1.0 \mathrm{~cm}$.

suspension also improved the infection rate in a Chinese soybean variety (Liu et al. 2008). In this study, 0, 0.02, and $0.04 \%$ of Silwet L-77 were added to the Agrobacterium suspension, and addition of $0.02 \%$ of Silwet L-77 resulted in optimal transformation efficiency for Kariyutaka. Ten scratches on the cotyledonary node section and application of $0.02 \%$ of Silwet L-77 to Agrobacterium suspension yielded the highest Agrobacterium-infection rate with normal formation of adventitious shoots (Figure 1E). The shoots were subsequently transferred to a rooting medium with $6 \mathrm{mg}^{-1}$ of glufosinate-ammonium. We found that some adventitious shoots were resistant to the glufosinateammonium (Figure $1 \mathrm{~F}$ ). Young $\mathrm{T}_{0}$ plants formed flower buds immediately after transplantation to the soil (Figure $1 \mathrm{G})$, resulting in production of $T_{1}$ seeds within 3 months 
of transplantation to the soil (Figure 1H, I). In this study, we obtained $\mathrm{T}_{1}$ seeds 5 to 6 months after Agrobacterium infection.

Up to five $T_{0}$ plants were generated from each explant pre-wounded by 10 times of scratching. We assigned the pertinent $\mathrm{T}_{0}$ plant numbers (e.g., IG1-1 or IG2-1) and considered $\mathrm{T}_{0}$ plants from the same explant as clones. We have summarized the frequency in the transformation that was performed with a protocol presented in this study (Table 1). The frequency of generating independent $\mathrm{T}_{0}$ plants ranged from $8.0 \%$ to $14.8 \%$ with a mean $10.8 \%$ (relative number of independent $\mathrm{T}_{0}$ plants to number of explants infected) in this study (Table 1). Accordingly, the frequency of generating $\mathrm{T}_{0}$ plants with the modified method was up to approximately 3 -fold that in the original method (data not shown). In this study, we obtained $32 \mathrm{~T}_{0}$ plants in total and $24 \mathrm{~T}_{0}$ plants set seeds (Table 1). These seeds were sowed and $T_{1}$ plants were subsequently grown. The existence and expression of the Bar gene were estimated from applying Basta onto leaves of the $T_{1}$ seedling (Figure 2A). In addition to resistant test in applied Basta, a total of $18 \mathrm{~T}_{1}$ plants from $4 \mathrm{~T}_{0}$ plants (IG2-1, IG6-1, IG13-1, and IG40-1) was characterized by PCR analysis using the specific primers for the Bar gene, Southern blot analysis using the specific probe for the intron GUS gene, and a GUS histochemical assay. The intact region of T-DNA was integrated into the soybean genome and the intron GUS gene was stably expressed in all 16 resistant $T_{1}$ plants to applied Basta (Figure 2B). These results suggested that the resistance to Basta applied in $T_{1}$ seedlings was available to ascertain the transmission of transgene into the $T_{1}$ plants. The integration of the intron GUS gene was also confirmed in the other Basta-resistant $\mathrm{T}_{1}$ plants. This analysis indicated that approximately $40 \%(13 / 32)$ of the $\mathrm{T}_{0}$ plants stably transmitted transgenes to the $\mathrm{T}_{1}$ plants (Table 1). The transformation frequency based on the relative number of stable $T_{0}$ transgenic plants to number of explants ranged from $3.6 \%$ to $5.6 \%$ with a mean $4.4 \%$ (Table 1). The transformation frequency in the method presented in this study was 4 times as high as in that obtained with our previous method (Sato et al. 2007). Furthermore, the frequency was comparable to the highest efficiency $(3.8 \%)$, which was expressed with the same definition as us, in a previous soybean transformation examined by Paz et al. (2006). The test of resistance to Basta applied onto $T_{1}$ seedlings also revealed the existence of unstable $\mathrm{T}_{0}$ plants on the transmission of transgenes to their $T_{1}$ progenies. A total of $11 \mathrm{~T}_{0}$ plants failed to transmit the Basta-resistance into $T_{1}$ progenies, although these $T_{0}$ plant set seeds (Table 1). Because these unstable $\mathrm{T}_{0}$ plants might be chimeric plants or undergo gene silencing, it is necessary to confirm the integration of the Bar gene or intron GUS

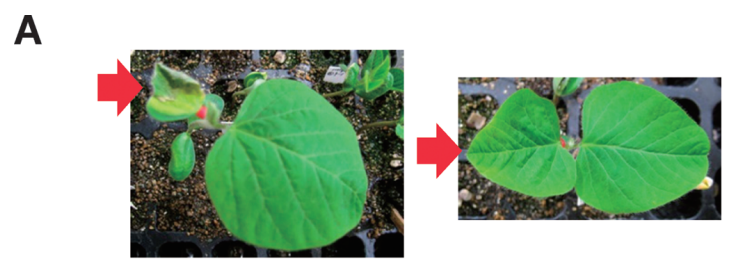

\section{B}
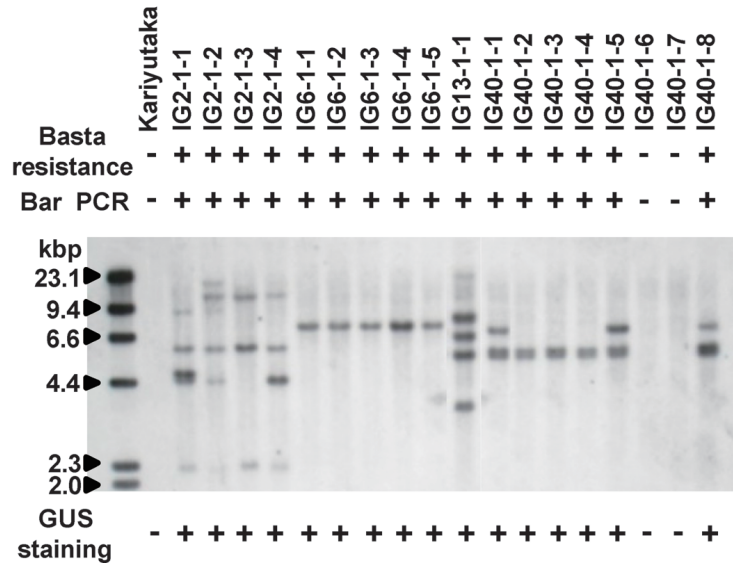

Figure 2. Characterization of $T_{1}$ plants. (A) Sensitive (left) and resistant (right) $T_{1}$ seedlings to applied Basta. Red arrows indicate leaves applied with Basta which were diluted 1,000-fold. (B) Resistance test to applied Basta, PCR analysis using specific primers for the Bar gene, Southern blot analysis using a specific probe to the intron GUS gene, and GUS histochemical assay in Kariyutaka and 18 $\mathrm{T}_{1}$ plants from $4 \mathrm{~T}_{0}$ plants (IG2-1, IG6-1, IG13-1, and IG40-1). Plant identification number of $\mathrm{T}_{1}$ plant was indicated at the top of each lane. Basta-resistance is expressed as + (resistant) or - (sensitive) at the top of each lane. Integration of the Bar gene is expressed as + (positive) or - (negative) for PCR analysis at the top of each lane. Southern blot analysis was conducted by digesting approximately $10 \mu \mathrm{g}$ of genomic DNA with the restriction enzyme $X b a$ I and hybridization with the specific probe mentioned above. The size of the molecular weight marker is indicated on the left side of a panel. GUS histochemical assay is expressed as + (positive) or - (negative) at the bottom of each lane. Two $T_{1}$ plants, IG40-1-6 and IG40-1-7, from IG40-1 showed negative results for all analyses.

Table 1. Transformation frequency of the method presented in this study

\begin{tabular}{|c|c|c|c|c|c|c|}
\hline \multirow{2}{*}{$\begin{array}{c}\text { Total number of } \\
\text { explants }^{\mathrm{a}}\end{array}$} & \multicolumn{2}{|c|}{ Explants generating $\mathrm{T}_{0}$ plant } & \multicolumn{2}{|c|}{ Explants generating fertile $T_{0}$ plants $^{\mathrm{c}}$} & \multicolumn{2}{|c|}{ Explants generating stable $\mathrm{T}_{0}$ plants $^{\mathrm{d}}$} \\
\hline & Total number ${ }^{\mathrm{a}}$ & Percentage $^{\mathrm{b}} \pm \mathrm{SD}$ & Total number ${ }^{\mathrm{a}}$ & Percentage $^{b} \pm S D$ & Total number ${ }^{\mathrm{a}}$ & Percentage $^{\mathrm{b}} \pm \mathrm{SD}$ \\
\hline 292 & 32 & $10.8 \pm 3.6$ & 24 & $8.0 \pm 3.5$ & 13 & $4.4 \pm 1.1$ \\
\hline
\end{tabular}

a Total number was obtained from three replicate transformation experiments.

Transgenes were confirmed by a resistant test to applied Basta and/or PCR analysis using specific primers for the intron GUS or the Bar genes.

${ }^{\mathrm{b}}$ Percentage represents mean of the relative number of explants generating $\mathrm{T}_{0}$ plants to number of explants used in three replicate experiments.

${ }^{\mathrm{c}}$ Fertile $\mathrm{T}_{0}$ plants indicate plants which were setting seeds.

${ }^{\mathrm{d}}$ Stable $\mathrm{T}_{0}$ plants indicate $\mathrm{T}_{0}$ plants transmitting resistance to applied Basta into $\mathrm{T}_{1}$ progenies. 


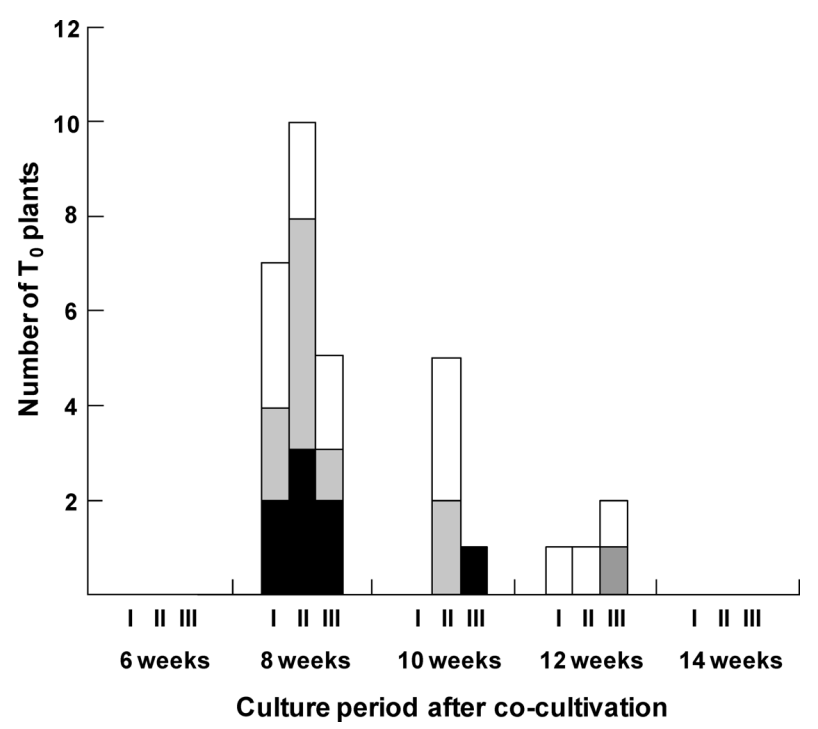

Figure 3. Number of $\mathrm{T}_{0}$ plants generated at $6,8,10,12$, and 14 weeks after starting the co-cultivation. White, gray, and black columns indicate stable (plants transmitting Basta-resistance to the $T_{1}$ progenies), unstable (plants not transmitting Basta-resistance), and sterile $\mathrm{T}_{0}$ plants, respectively. I, II, and III indicate three replicate transformation experiments with the method presented in this study.

gene in these $T_{1}$ progenies. Stable $T_{0}$ plants were generated at the late stage of shoot elongation. Three $\mathrm{T}_{0}$ plants out of 4 , i.e. $75 \%$ of all $\mathrm{T}_{0}$ plants, which were generated at 12 weeks after co-cultivation, possessed stable transgene(s) that were transmitted to their $\mathrm{T}_{1}$ progenies, although only $7 \mathrm{~T}_{0}$ plants out of 22 , i.e. $32 \%$ of all $\mathrm{T}_{0}$ plants, which were generated at 8 weeks after the co-cultivation, stably transmitted the Basta-resistance to their $\mathrm{T}_{1}$ progenies (Figure 3). Therefore, promoting the formation of adventitious shoots at 12 weeks after the co-cultivation also might lead to a higher frequency of stable transgenic plants.

The transformation frequency of soybean was significantly increased with the method presented in this study. These improvements also might be applied to increase of transformation frequency in other methods of soybean transformation. An efficient and rapid transformation protocol is a key requirement for functional genomics. We hope that the transformation method described in this report will contribute to future studies in the molecular genetics of soybean.

\section{Acknowledgements}

We thank Yasuko Kitsui for technical assistance and Dr. Ishimoto (National Agriculture Research Center for Hokkaido Region) for helpful suggestions. This work was supported in part by Grantsin-Aid for Scientific Research from the Ministry of Education, Culture, Sports, Science and Technology of Japan.

\section{References}

Birch RG (1997) Plant transformation: Problem and strategies for practical application. Annu Rev Plant Physiol Mol Biol 48: 297-326

Curtis MD, Grossniklaus U (2003) A gateway cloning vector set for high-throughput functional analysis of genes in planta. Plant Physiol 133: 462-469

Dang W, Wei Z (2007) An optimized Agrobacterium-mediated transformation for soybean for expression of binary insect resistance genes. Plant Sci 173: 381-389

Dinkins RD, Collins GD (2008) Agrobacterium-mediated genetic transformation of soybean. In: Kirti PD (ed) Handbook of new technologies for genetic improvement of legumes. CRC Press, Florida, pp 89-102

Hinchee MAW, Conner-Ward DV, Newell CA, McDonnell RE, Sato SJ, Gasser CS, Fischhoff DA, Re DB, Fraley RT, Horsch RB (1998) Production of transgenic soybean plants using Agrobacterium-mediated DNA transfer. BioTechnol 6: 915922

Liu S-J, Wei Z-M, Huang J-Q (2008) The effect of co-cultivation and selection parameters on Agrobacterium-mediated transformation of Chinese soybean varieties. Plant Cell Rep 27: 489-498

Meurer CA, Dinkins RD, Collins GB (1998) Factors affecting soybean cotyledonary node transformation. Plant Cell Rep 18: $180-186$

Niwa Y, Hirano T, Yoshimoto K, Shimizu M, Kobayashi H (1999) Non-invasive quantitative detection and applications of nontoxic, S65T-type green fluorescent protein in living plants. Plant J 18: 455-463

Ohta S, Mita S, Hattori T, Nakamura K (1990) Construction and expression in tobacco of a $\beta$-glucuronidase (GUS) reporter gene containing an intron within the coding sequence. Plant Cell Physiol 31: 805-813

Olhoft PM, Lin K, Galbraith J, Nielsen NC, Somers DA (2001) The role of thiol compounds in increasing Agrobacteriummediated transformation of soybean cotyledonary-node cells. Plant Cell Rep 20: 731-737

Paz MM, Martinez JC, Kalvig AB, Fonger TM, Wang K (2006) Improved cotyledonary node method using an alternative explant derived from mature seed for efficient Agrobacterium-mediated soybean transformation. Plant Cell Rep 25: 206-213

Sato H, Yamada T, Kita Y, Ishimoto M, Kitamura K (2007) Production of transgenic plants and their early seed set in Japanese soybean variety, Kariyutaka. Plant Biotechnol 5: 533-536

Trick HN, Finer JJ (1997) SAAT: Sonication-assisted Agrobacterium-mediated transformation. Transgenic Res 6: 329-336

Xue R-G, Xie H-F, Zhang B (2006) A multi-needle-assisted transformation of soybean cotyledonary node cells. Biotechnol Lett 28: 1551-1557

Zeng P, Vadnais DA, Zhang Z, Polacco JC (2004) Refined glufosinate selection in Agrobacterium-mediated transformation of soybean [Glycine max (L.) Merrill]. Plant Cell Rep 22: $478-482$ 